\title{
Electron Cyclotron interaction in the early stage of EC-assisted plasma breakdown
}

\author{
Daniela Farina ${ }^{1, \star}$ \\ ${ }^{1}$ Istituto di Fisica del Plasma, Consiglio Nazionale delle Ricerche, Milano, Italy
}

\begin{abstract}
Electron Cyclotron (EC) wave interaction with very low temperature electrons in conditions like those occurring for EC-assisted plasma breakdown is investigated via the Hamiltonian adiabatic formalism. Estimates of interest for applications to present tokamak experiments are derived. The analysis allows ultimately to derive analytically the particle energy gain per single beam crossing and to characterize the dependency of the maximum possible energy variation as a function of the wave frequency, harmonics and polarization and EC power and beam width. The investigation confirms that electrons can easily gain energies well above the ionization energy in most conditions, as observed in experiments.
\end{abstract}

\section{Introduction}

As pointed out long ago in the literature [1], wave interaction with an electron crossing the microwave beam in single transit is in general non linear in the initial stage of EC pre-ionization. In the very first phase of a plasma discharge with EC-assisted breakdown, the electron motion can be described as single particle in free space under the action of a localized e.m. beam in a static magnetic field. The EC beam is assumed to be injected into the plasma with a Gaussian-like profile such that wave electric field amplitude is $E=E_{M} \exp \left(-r^{2} / w^{2}\right)$, with $r$ the beam radius, $w$ the waist and $E_{M}$ its maximum value. For a magnetized electron at room temperature $\left(T_{e}=0.03 \mathrm{eV}\right)$ and for EC beam parameters typical of ITER $(P=1 \mathrm{MW}$, width $w=0.05-0.1 \mathrm{~m}, f=170 \mathrm{GHz}$ ) as well as of present experiments, the interaction regime is characterized by $\tau_{c y c l} \ll$ $\tau_{\text {trap }} \ll \tau_{\text {flight }} \ll \tau_{\text {coll }}$, with $\tau_{\text {cycl }} \simeq 6 \times 10^{-12} \mathrm{~s}, \tau_{\text {trap }} \approx 10^{-8}$ $\mathrm{s}, \tau_{\text {flight }} \approx 1-5 \times 10^{-6} \mathrm{~s}, \tau_{\text {coll }} \approx 5 \times 10^{-4}-5 \times 10^{-3} \mathrm{~s}$, the cyclotron period, the trapping time in the wave field, the time flight in the beam and the collision time, respectively. Being $\tau_{\text {trap }}$ much shorter than the time scale of the variation of the e.m. wave amplitude, the above conditions define an adiabatic nonlinear regime (widely investigated in the past, see e.g. [1-4]). This regime can be described theoretically via a Hamiltonian formalism, making use of the adiabatic invariants. A rigorous identification of the relevant elementary physical processes can then be performed within this approach.

\section{Hamiltonian electron motion}

The motion of an electron interacting with an EC wave with frequency $\omega$, propagating perpendicularly to the static

\footnotetext{
${ }^{\star}$ Corresponding author: farina@ifp.cnr.it
}

uniform magnetic field $\mathbf{B}_{0}=B_{0} \mathbf{e}_{z}$, can be described in the Coulomb gauge (with zero scalar potential) by the Hamiltonian function $H(\mathbf{x}, \mathbf{P})=m c^{2} \gamma=m c^{2}[1+\mid \mathbf{P} / m c+$ $\left.e /\left.c \mathbf{A}\right|^{2}\right]^{1 / 2}$, where $\mathbf{P}=\mathbf{p}-e / c \mathbf{A}$ is the canonical momentum conjugate to $\mathbf{x}, \mathbf{A}(\mathbf{x}, t)=\mathbf{A}_{0}(\mathbf{x})+\mathbf{A}_{1}(\mathbf{x}, t)$ is the vector potential, sum of a static and e.m. part. In the following normalized variables are used, i.e., time, length, momentum, energy and Hamiltonian, and vector potential over $\Omega^{-1}, c \Omega^{-1}, m c, m c^{2}$, and $e / m c^{2}$, respectively being $\Omega=e B / m c$ the cyclotron frequency. The static and e.m. vector potentials can be written as $\mathbf{A}_{0}=-y \hat{\mathbf{x}}, \mathbf{A}_{1}=$ $\epsilon(\mathbf{x})\left[e_{x} \cos \chi \hat{\mathbf{x}}+e_{y} \sin \chi \hat{\mathbf{y}}+e_{z} \cos \chi \hat{\mathbf{z}}\right]$, being $\epsilon=e E / m c \omega$ the normalized amplitude, $\chi=v(N x-t)$ the phase, $\mathbf{e}=\mathbf{E} / E$ the wave polarization vector, $N$ the refractive index, and $v=\omega / \Omega$ the frequency ratio.

Following the approach described in [3], a new timeindependent Hamiltonian with two degrees of freedom, valid for $\omega$ close to a cyclotron harmonic $n \Omega$, and at first order in the wave amplitude $\epsilon \ll 1$ can be obtained via canonical transformations

$$
H\left(z, \theta, P_{z}, I\right)=\gamma-v_{n} I=H_{0}\left(P_{z}, I\right)+H_{1}\left(z, \theta, P_{z}, I\right)
$$

with $v_{n}=v / n=\omega / n \Omega, H_{0}\left(P_{z}, I\right)=\Gamma-v_{n} I$, and $H_{1}\left(z, \theta, P_{z}, I\right)=\epsilon(z) \Theta_{n}\left(P_{z}, I\right) / \Gamma \cos n \theta$, where $I$ and $\theta$ are action-angle variables, $P_{z}$ is the parallel canonical momentum conjugate to $z$, and $\Gamma=\left(1+2 I+P_{z}^{2}\right)^{1 / 2}$. For the unperturbed system $(\epsilon=0), \theta$ is the phase of the gyromotion, $I=p_{\perp}^{2} / 2$, and $P_{z}=p_{z}$. The polarization term $\Theta_{n}$ reads $\Theta_{n}=\sqrt{2 I}\left[e_{-} J_{n-1}(b)+e_{+} J_{n+1}(b)\right]+P_{z} e_{z} J_{n}(b)$, with $e_{ \pm}=\left(e_{x} \pm e_{y}\right) / 2$ and $b=v N \sqrt{2 I}$.

In Figure 1, two examples of solution of the Hamiltonian equations of motion are shown for ITER parameters, second harmonic extraordinary mode (XM) polarization. The initial conditions refer to thermal electrons at $T_{e}=0.03 \mathrm{eV}$, just outside the beam region, i.e., at $t=0$, 
$z_{0}=-4 w(w=0.1 \mathrm{~m}), P_{z 0}=\beta_{t h}$ and $I_{0}=\beta_{t h}^{2} / 2$ being $\beta_{t h}=v_{t h} / c=\left(T_{e} / m c^{2}\right)^{1 / 2}$ the thermal velocity normalized to the speed of light. In the two cases, which differ in the initial phase value $\theta$ only, two quite distinct final energy values are obtained, in one case the electron recovers its initial energy value while in the other one it gains a large amount of energy as a consequence of beam crossing. While the electron crosses the EC beam, it becomes non linearly trapped in the wave field for $\epsilon=\epsilon_{s} \simeq 0.6 \epsilon_{M}$ (i.e., $|z| \simeq 0.1 \mathrm{~m}$ ), and then its energy experiences large variations, getting the maximum value at $z \sim 0$ (up to $\sim 180$ $\mathrm{eV}$ ). When the e.m. field decreases (for $z>0$ ) the electron becomes untrapped (for the same $\epsilon_{s}$ value) and exits the beam region either with final energy $\approx 0.03 \mathrm{eV}$ (blue dotted curve) or $\simeq 60 \mathrm{eV}$ (red solid curve), much larger than the initial one. For the chosen parameters, the time required to travel the distance $\Delta z=2\left|z_{0}\right|$ is $\approx 8 \mu \mathrm{s}$. In contrast to the perpendicular energy large variations, the parallel canonical momentum $P_{z}$ shown in Fig. 2 is almost constant while the electron crosses the beam region, with maximum variation of the order of $\sim 3 \%$ and negligible final net variation $(\sim 0.2 \%)$.

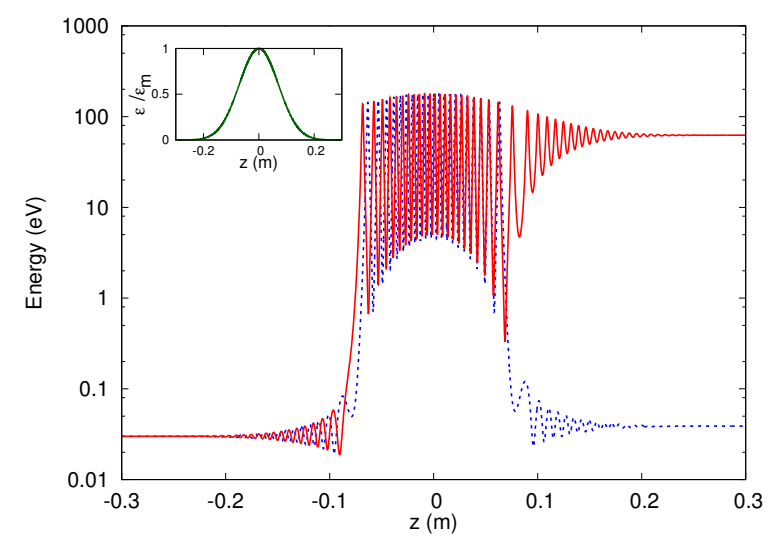

Figure 1. Electron energy $W=m c^{2}(\gamma-1)$ (logarithmic scale) versus coordinate $z$ for two different initial phase values $\theta$. For reference, the electric field amplitude versus $z$ is shown in the inset plot on the top left. The chosen parameters are $P=1 \mathrm{MW}$, beam waist $w=0.1 \mathrm{~m}$, XM second harmonic, $1-v_{n}=6 \times 10^{-5}$.

A peculiar behaviour of the system is reported in Figure 3, where the evolution of the action is shown for various input power keeping the other parameters constant. The same quite large net action variation is found independently of the e.m. input power, provided that the electron becomes trapped in the wave field. The threshold power for trapping is $\sim 250 \mathrm{~kW}$ for the considered beam parameters and initial particle energy, and electrons recover their initial energy for all phase values in the lowest power case for which trapping does not occur. Quite similar results are found at first harmonic, but with a much larger energy variation (see below).

\section{Adiabatic nonlinear theory}

The electron motion is governed by the Hamiltonian function (1), and is characterized by a couple of fast action-

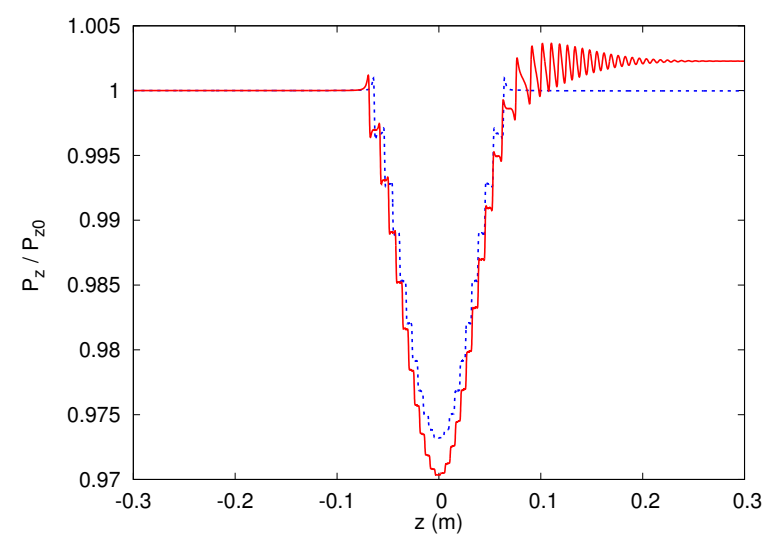

Figure 2. Parallel canonical momentum versus coordinate $z$ for the same cases of Fig. 1.

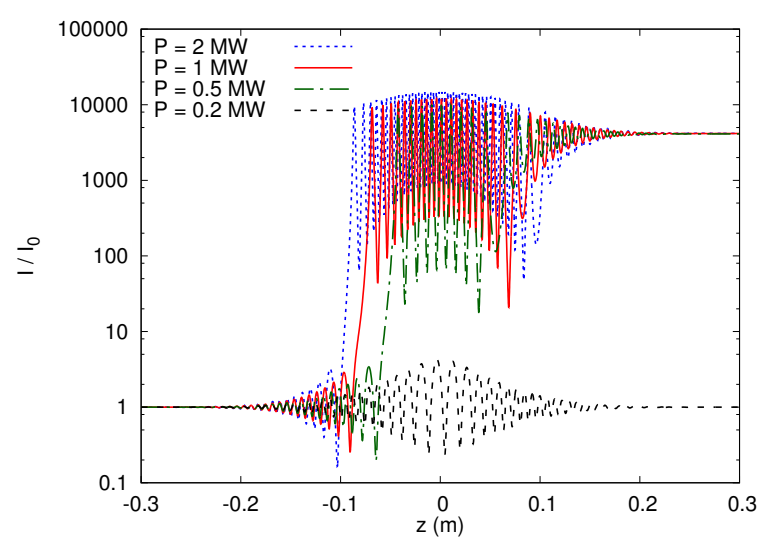

Figure 3. Evolution of canonical action $I$ normalized to initial values as a function of coordinate $z$ for various input EC power, $P=0.2,0.5,1,2 \mathrm{MW}$. Other parameters as in Fig. 1 .

angle variables $(\theta, I)$ and of slow canonically conjugate variables $\left(z, P_{z}\right)$ in the considered regime of variation of the e.m. field. Whenever the e.m. field variation experienced by the electron while crossing the beam is sufficiently slow, the action integral $J\left(z, P_{z}\right) \equiv n / 2 \pi \oint I d \theta$ is an adiabatic invariant of the motion, that breaks only at separatrix crossing between trapped and untrapped orbits. Breaking of the invariant via trapping/untrapping allows to connect different regions in phase space and provides the mechanism for net energy variation. This physics process for EC nonlinear interaction was investigated in the past in [1-4].

The basic process can be described recalling that the initial and final states are determined by the unperturbed Hamiltonian $H_{0}=$ const (valid for particles outside the e.m. illumination region), with initial and final states related by $\Delta \gamma=v_{n} \Delta I$, and $\Delta P_{z}^{2}=\Delta \gamma\left[\Delta \gamma-2\left(1 / v_{n}-\gamma_{0}\right)\right]$. In addition, $\Delta P_{z} \simeq 0$ is satisfied under adiabatic conditions in perpendicular propagation. A particle with initial $I_{0}$ and $P_{z 0}$ belonging initially to a given branch of the Hamiltonian will enter the beam region and eventually cross the separatrix for $\epsilon=\epsilon_{s}$ for increasing $\epsilon$ (if $\epsilon_{s} \leq \epsilon_{M}$ ), being trapped and then untrapped for decreasing $\epsilon$ for $\epsilon=\epsilon_{s}$, and 
will exit the beam region with final values $I_{f}$ and $P_{z f}$, with $P_{z f} \simeq P_{z 0}$, within the present approximation. A net energy variation can occur only if $H_{0}$ is double valued in action $I$, condition satisfied if $\omega<n \Omega$ and the resonant condition $\partial H_{0} / \partial I=0$ is met for an action value $I_{r}$ corresponding to the unperturbed resonant action at constant $P_{z}$.

In Figure 4, the interaction process is sketched in the phase space of the fast variables for increasing $\epsilon$ values: motion across the EC beam corresponds to snapshots from top left to low right and viceversa, i.e., to first increasing and decreasing $\epsilon$ values. The particle will lie initially on either the lower or the upper branch depending if $I_{r}$ is larger or smaller than $I_{0}$ (see upper left snapshot). At an intermediate $\epsilon$ value not shown here, the two branches merge, and the particle is nonlinearly trapped (snapshots in lower row). After beam crossing both branches can be populated. Note that $\Delta \gamma$ will be different from zero only if the particle after the last separatrix crossing will not lie on the same branch of the Hamiltonian as initially.
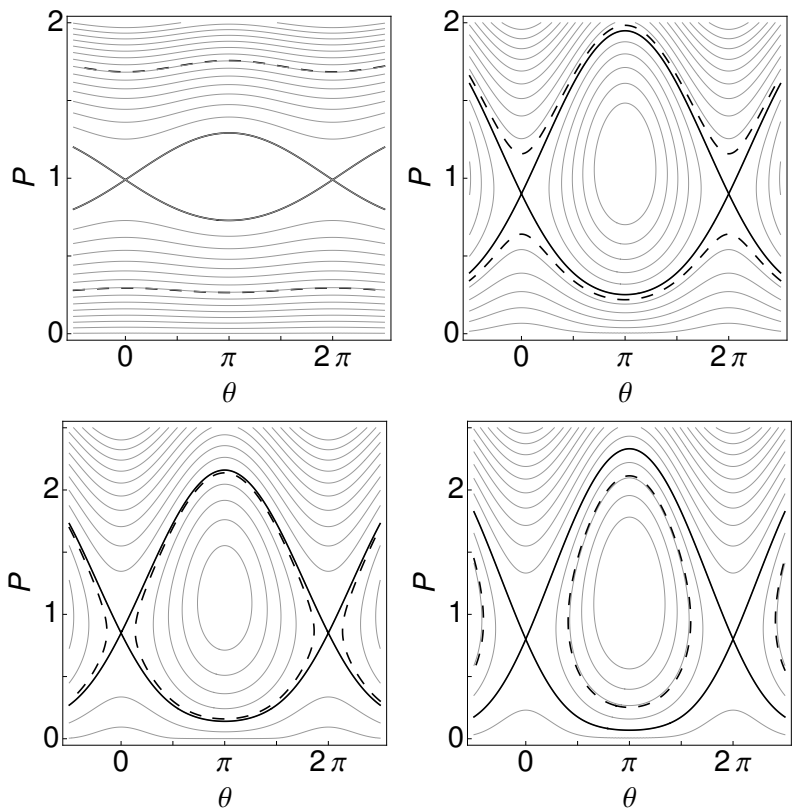

Figure 4. Snapshots of phase space of the fast variables for increasing value of the local e.m. field $\epsilon$ (from top left to bottom right). Variable $P$ is the action variable normalised over the resonant value. Black solid line is the separatrix, dashed line is the level curve corresponding to the initial Hamiltonian value.

The evolution of the slow variable $P_{z}$ in the various stage of the motion is described via the constancy of the adiabatic invariant $J$, i.e., $J\left(\epsilon, P_{z}\right)=I_{0}$ for $\epsilon \leq \epsilon_{s}$, $J\left(\epsilon, P_{z}\right)=J_{s}$ for $\epsilon_{s} \leq \epsilon$, and $J\left(\epsilon, P_{z}\right)=I_{f}$ for $\epsilon \leq \epsilon_{s}$, being $J_{s}=A_{s} / 2 \pi$ with $A_{s}$ the area of the phase space region inside the separatrix at its crossing. Being the motion adiabatic in each stage, the global variation of the action variable $I$ is equal to the jump of the adiabatic integral at the separatrix

$$
\Delta \gamma=v_{n}\left(I_{f}-I_{0}\right)=v_{n}\left(\left|J_{f}\left(\epsilon_{s}, P_{z s}\right)\right|-\left|J_{0}\left(\epsilon_{s}, P_{z s}\right)\right|\right),
$$

where $P_{z s}$ is the $P_{z}$ value at the separatrix, and $J_{0, f}\left(\epsilon, P_{z}\right)$ is the action integral in the initial and final stage of the motion, i.e., before and after the separatrix crossings. The $\epsilon$ and $P_{z}$ values at the separatrix are related to the initial (or final) momentum values by means of the following system of equations

$$
\begin{array}{r}
I_{0, f}=\left|J_{0, f}\left(\epsilon_{s}, P_{z s}\right)\right|, \\
H_{0}\left(I_{0, f}, P_{z 0, f}\right)=H_{s}\left(\epsilon_{s}, P_{z s}\right),
\end{array}
$$

where $H_{s}$ is the Hamiltonian computed at the separatrix.

As shown in Fig. 3, the energy variation is independent on the peak value of the e.m. field $\epsilon_{M}$, provided trapping occurs. The maximum field amplitude determines the set of initial momenta for which an energy transition can occur: $\epsilon_{s}\left(I_{0}, P_{z 0}\right) \leq \epsilon_{M}$. The above expressions for the momentum variation in case of trapped motion can be computed explicitly in the weakly-relativistic approximation, that is well justified for the electron energies in the physical regimes of interest here.

In the following, the analysis of the first two harmonics $n=1,2$ is briefly summarized (see, e.g., [1, 3, 4] for detailed derivations). Expanding Eq. (1) for small energy values up to second order in the action in $H_{0}$, and to lowest order in the polarization term, one obtains

$$
\begin{gathered}
H_{0}=P_{z}^{2} / 2+I_{r}\left(P_{z}\right) I-I^{2} / 2, \\
H_{1}=\epsilon(z) T_{n}\left(P_{z}\right)[2 I]^{n / 2} \cos n \theta
\end{gathered}
$$

with $I_{r}\left(P_{z}\right)=1-v_{n}-P_{z}^{2} / 2$ the resonant action, and $T_{n}\left(P_{z}\right)=(N v)^{n-1}\left[\left(e_{x}-e_{y}\right)^{q}+\left(e_{z} N v_{n} P_{z}\right)^{1-q}\right] /\left[2^{n}(n-1) !\right]$, with $q=0,1$ for OM, XM.

To determine the separatrix crossing and compute the adiabatic invariants it is convenient to investigate the Hamiltonian (5) in the phase space of the fast variables for fixed $\epsilon$ and $P_{z}$. The singular points $\left(\theta_{p}, I_{p}\right)$ of the Hamiltonian $H=H_{0}+H_{1}$ are the solutions of the set of equations $\partial H / \partial I=0, \partial H / \partial \theta=0$. In the resonant case $\left(I_{r}>0\right.$, i.e., $P_{z}^{2} / 2<1-v_{n}$ and $\left.v_{n}<1\right)$, a separatrix is found for $\epsilon \leq \epsilon_{c n}=\beta_{c n} I_{r}^{2-n / 2} / T_{n}$, with $\beta_{c 1}=(2 / 3)^{3 / 2}$ and $\beta_{c 2}=1 / 2$. In the resonant case, the phase space topology is pendulum-like for low $\epsilon$ values, while it shows quite different features for $\epsilon \lesssim \epsilon_{c n}$. The explicit expressions of the adiabatic integrals $J_{n l}$ and $J_{n u}$ computed on the lower and upper branch of the separatrix are reported in [3], the relation $J_{n l}+J_{n u}=2 I_{r}\left(P_{z s}\right)$ being valid for $n=1,2$.

Let us consider a very low energy particle with initial momentum $I_{0}, P_{z 0}$ outside the EC beam region, flying towards it, with initial action $I_{0}$ lower than the resonant action $I_{r}$. The separatrix crossings are determined by Eqs. $(3,4)$, in the first crossing one has $J_{n l}=I_{0}$, while in the last one either $J_{n l}=I_{f}$ or $J_{n u}=I_{f}$, depending on the final branch of the Hamiltonian, lower or upper. In the first case $I_{f}=I_{0}$, so the electron recover its initial energy $(\Delta \gamma=0)$, while in the last case it experiences a net energy variation

$$
\Delta \gamma=v_{n}\left(J_{n u}\left(\epsilon_{s}, P_{z s}\right)-J_{n l}\left(\epsilon_{s}, P_{z s}\right)\right)=2 v_{n}\left[I_{r}\left(P_{z s}\right)-I_{0}\right]
$$

Equation (6) clearly shows that the nonlinear energy variation due to trapping in the wave field depends on the initial energy and frequency mismatch only. Moreover, for a given set of launching parameters the maximum energy gain occurs for particle with the lowest possible energy. On 

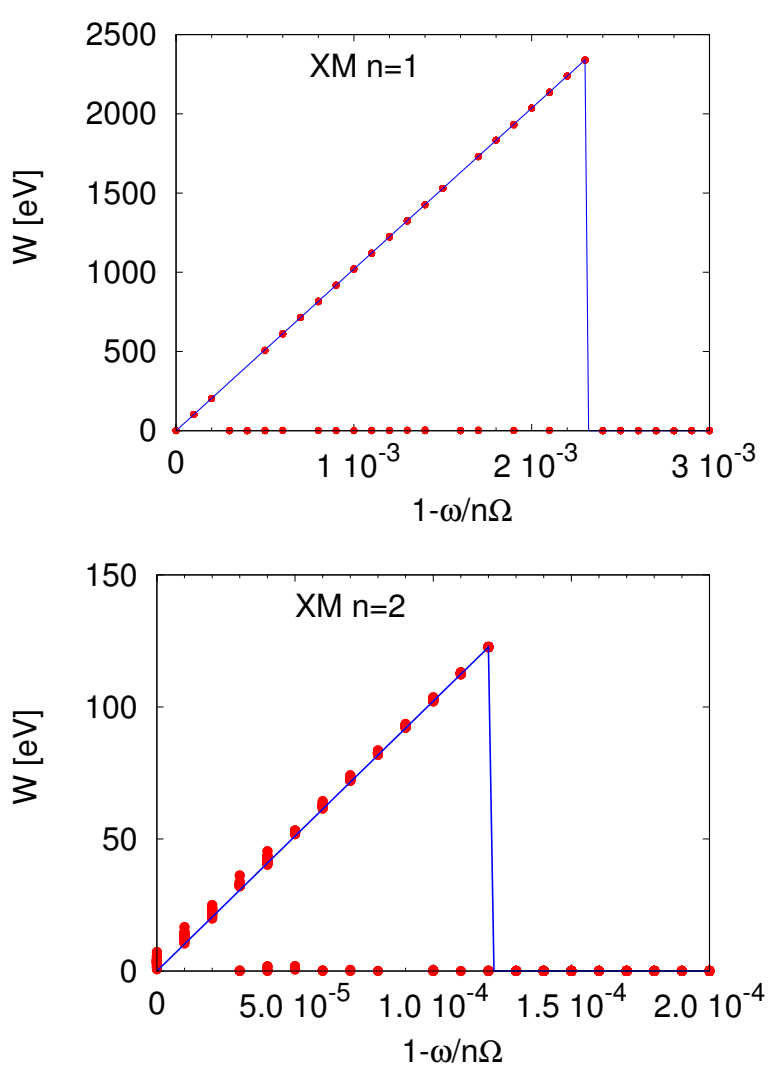

Figure 5. Electron final energy $W$ as a function of the EC frequency ratio for $P=1 \mathrm{MW}, w=10 \mathrm{~cm}, f=170 \mathrm{Ghz}$ and XM1, XM2 perpendicular propagation. Solid blue curve correspond to the analytical estimate (7) of the maximum energy, red points to the numerical solution of Hamiltonian equations (50 uniformly distributed $\theta_{0}$ initial values with $I_{0}=\beta_{t h}^{2} / 2, P_{z 0}=\beta_{t h}$ are evaluated for each frequency ratio). Note the different scales for the two harmonics.

the contrary, the maximum field amplitude $E_{M}$ determines under which conditions trapping occurs.

Simple analytical estimates for the energy variation can be derived for electrons with very low initial energy. The analysis demonstrates that $P_{z s} \simeq P_{z 0}$ and $I_{r}\left(P_{z s}\right) \simeq$ $I_{r}\left(P_{z 0}\right)=1-v_{n}-P_{z 0}^{2} / 2$, so that

$$
\Delta \gamma \simeq 2 v_{n}\left(1-v_{n}-I_{0}-P_{z 0}^{2} / 2\right) \text {. }
$$

Figure 5 shows the very good agreement between the analytical estimate (7) and the results obtained from the solution of the Hamiltonian equations of motion (as in Sec. 2) for various initial phase values and same thermal initial action $I_{0}$ and $P_{z 0}$. The energy increases linearly with $1-v_{n}$, i.e. with the frequency mismatch from the resonance, up to a maximum value which depends on the maximum field amplitude $\epsilon_{M}$, and drops to zero afterwards. Since very low energy particles cross the separatrix for $\epsilon \simeq \epsilon_{c n}$, a simple estimate of the maximum energy $W=m c^{2}(\gamma-1)$ for increasing $1-v_{n}$ is obtained by imposing $\epsilon_{M}=\epsilon_{c n}$

$$
W_{\text {max }} \simeq 2 m c^{2}\left[\epsilon_{M} P_{z}^{1-q} /\left(2 \beta_{c n}\right)\right]^{2 /(4-n)}
$$

The scaling of the maximum energy $W_{\max }$ with the injected EC beam parameters is obtained recalling that $\epsilon_{M} \propto P^{1 / 2} /(f w)$. In case of the X-mode one has

$$
\begin{gathered}
W_{\text {max }, X M 1} \simeq 15.6 P^{1 / 3} /\left(f^{2 / 3} w^{2 / 3}\right), \\
W_{\text {max }, X M 2} \simeq 2.1 P^{1 / 2} /(f w),
\end{gathered}
$$

where $W, P, f, w$ are measured in $\mathrm{keV}, \mathrm{MW}, \mathrm{GHz}$ and meters, respectively. The above estimates are valid in the considered low density cold plasma. Note that the EC wave energy transfer to cold electrons is much less efficient in case of the OM, due to the different scaling of the polarization term

\section{Conclusions}

The analysis performed here provides a theoretical description of the EC wave-particle non linear interaction in the pre-ionization phase (much before collisions can play a role). Simple analytical estimates, in very good agreement with numerical particle simulations, are derived for the maximum energy gain as a function of the peak electric field amplitude, and expressed in terms of the EC power, frequency and beam spot size. The results confirm that cold electrons can easily gain energies well above the ionization energy in most conditions, as observed in many experimental conditions $[5,6]$.

The work leading to this publication has been funded partially by Fusion for Energy under Grant F4E-615. This publication reflects the views only of the author, and Fusion for Energy cannot be held responsible for any use which may be made of the information contained therein.

\section{References}

[1] E. V. Suvorov, M. D. Tokman, Sov. J. Plasma Phys., 14, 557 (1988)

[2] W. M. Nevins, T. D. Rognlien, and B. I. Cohen, Phys. Rev. Lett., 59, 60 (1987)

[3] D. Farina, R. Pozzoli, Phys. Fluids B, 3, 1570 (1991)

[4] I. A. Kotelnikov, G. V. Stupakov, Phys. Fluids B 2, 881 (1990); J. Plasma Phys., 45, 19 (1991).

[5] G. L. Jackson, et al., Nuclear Fusion, 47, 257 (2007)

[6] G.Granucci, et al., Nuclear Fusion, 55, 093025 (2015) 